\title{
EVALUACIÓN DE LA VEGETACIÓN DEL ESTRATO HERBÁCEO DE UN BOSQUE DEL ESPINAL SANTAFESINO (ARGENTINA)
}

\author{
Pedro A. Capelino \& Adrián G. Bender
}

Facultad de Ciencias Agrarias, Universidad Nacional del Litoral, Kreder 2805, 3080 Esperanza, Santa Fe, Argentina; capelinopedro@hotmail.com (autor corresponsal).

Abstract. Capelino, P. A. \& A. G. Bender. 2020. Evaluation of the vegetation of the herbaceous stratum in a forest of Espinal Santafesino (Argentina). Darwiniana, nueva serie 8(1): 23-41.

In the Espinal Santafesino only relicts of forests remain. The present study was carried out in the town of Sa Pereira, Argentina, in the herbaceous stratum of an algarrobo forest of approximately 100 ha. The aim was to perform a description of the herbaceous vegetation. Samples were taken in $16 \mathrm{~m}^{2}$ plots, using the Braun-Blanquet abundance-cover method (64 plots) on a base transect. Two samplings were performed, one in autumn-winter-spring (AWS) and another in spring-summer (SS). To characterize the herbaceous communities Simpson, Equity and Shannon diversity indexes were used. Three groups of herbaceous communities were identified: "flechillares", humid prairies and "gramillares", distinguishing eight variants thereof. There were 45 families and 156 species, with the most represented families Poaceae and Asteraceae ( 38 and 25 species respectively). On average, 20.8 and 18.1 species were recorded per plot for the AWS and SS samples. In the AWS and SS samples, an average Simpson diversity index of 6.72 and 8.42 was obtained, while the Shannon diversity index was 2.09 and 2.30, respectively. In the AWS samplings, the humid prairies were more diverse according to these indexes, followed by "flechillares" and "gramillares", whereas in the SS sampling, "flechillares" were the most diverse. The average equitability index for the AWS samples was generally lower than the SS for all the community groups.

Keywords. Floristic description; fodder; herbaceous plants; Santa Fe province; trees; vegetation relict.

Resumen. Capelino, P. A. \& A. G. Bender. 2020. Evaluación de la vegetación del estrato herbáceo de un bosque del Espinal Santafesino (Argentina). Darwiniana, nueva serie 8(1): 23-41.

En el Espinal Santafesino sólo quedan relictos de bosques. El presente estudio se llevó a cabo en la localidad de Sa Pereira, Argentina, en el estrato herbáceo de un bosque de algarrobos de aproximadamente 100 ha. El objetivo fue realizar una descripción de la vegetación herbácea. Los muestreos se realizaron en parcelas de $16 \mathrm{~m}^{2}$, utilizando el método de abundancia-cobertura de Braun-Blanquet (64 parcelas) sobre un transecto base. Se realizó un muestreo otoño-inverno-primaveral (OIP) y otro primaveroestival (PE). Para caracterizar a las comunidades herbáceas se utilizaron los índices de diversidad de Simpson, de Equitatividad y de Shannon. Se identificaron tres grupos de comunidades herbáceas: flechillares, praderas húmedas y gramillares, distinguiendo ocho variantes de las mismas. Se registraron 45 familias y 156 especies, siendo las familias más representadas Poaceae y Asteraceae (38 y 25 especies respectivamente). En promedio se registraron 20,8 y 18,1 especies por parcela para los muestreos OIP y PE. En los muestreos OIP y PE se obtuvo un índice de diversidad de Simpson promedio de 6,72 y 8,42; mientras que el índice de diversidad de Shannon fue de 2,09 y 2,30 respectivamente. En los muestreos OIP las praderas húmedas fueron más diversas según estos índices seguidos por flechillares y gramillares mientras que en los relevamientos $\mathrm{PE}$ los flechillares fueron los más diversos. El índice de equitatividad medio para los muestreos OIP fue en general menor que los PE para todos los grupos de comunidades.

Palabras clave. Árboles; descripción florística; forraje; plantas herbáceas; provincia de Santa Fe; relicto de vegetación. 


\section{INTRODUCCIÓN}

La provincia fitogeográfica del Espinal en la República Argentina se ubica entre los $28^{\circ}$ y $40^{\circ}$ de latitud $\mathrm{S}$, se caracteriza por su vegetación de bosque xerófito abierto con uno o dos estratos arbóreos de menos de diez metros de altura, un estrato arbustivo y otro herbáceo; además alternan con estos bosques palmares, sabanas y estepas de gramíneas (Cabrera, 1976; Atlas de los Bosques Nativos Argentinos, 2003). El distrito del Algarrobo representa una zona de transición entre la Pampa y el Chaco, ocupa el centro de las provincias de Santa Fe y Córdoba hasta la mitad Norte de la provincia de San Luis, sobre el sector Norte de la llanura chaco-pampeana. En este distrito quedan pocos relictos de bosques (Atlas de los Bosques Nativos Argentinos, 2003; Matteucci, 2012). La comunidad clímax es el bosque de algarrobo negro (Prosopis nigra) y de algarrobo blanco (Prosopis alba), mezclados con chañar (Geoffroea decorticans), y tala (Celtis tala), entre otros (Atlas de los Bosques Nativos Argentinos, 2003; Capelino et al., 2018).

Más allá de los variados beneficios materiales, ambientales y sociales brindados por los bosques y los pastizales, en la actualidad sólo quedan relictos de estos espacios en el Espinal Santafesino. Estos relictos se encuentran en su mayoría asociados a cursos de agua, ya que se ha avanzado sobre los lugares con mejores condiciones para el desarrollo de la agricultura (Lewis et al., 2006; Secretaría de Estado de Medio Ambiente y Desarrollo Sustentable, 2007; Matteucci, 2012). Asimismo, la explotación irracional del bosque sin un estudio y planificación previa ha favorecido su deterioro (Karlin \& Bernasconi, 2005; Matteucci, 2012). El desafío que se plantea al sector productivo y a los técnicos, es el de utilizar los ecosistemas sin desencadenar cambios en los suelos y la vegetación que alteren la capacidad de producción de forraje y de provisión de servicios ecológicos en forma irreversible. Para responder a ese desafío es necesario conocer a los bosques (Karlin \& Bernasconi, 2005) y los pastizales (Deregibus, 1988; Escobar, 2008) para realizar una producción ganadera, agrícola y forestal en forma sustentable.
Dentro de los antecedentes de estudios referidos el estrato herbáceo en el Espinal Santafesino se destacan las experiencias realizadas por Exner et al. (2004), Pensiero et al. (2005), Proyectos de Investigación Aplicada a los Recursos Forestales Nativos (2005) y Lewis et al. (2006) quienes hacen una descripción de la vegetación en las localidades de Esperanza, San Cristóbal, San Javier y Coronda. El objetivo general del presente trabajo es evaluar la vegetación del estrato herbáceo de un algarrobal del Espinal Santafesino. Los objetivos específicos son: describir las comunidades herbáceas, y determinar la abundancia y la cobertura de los componentes del estrato herbáceo para calcular índices de diversidad. La información generada permitirá conocer la vegetación cuantitativa y cualitativamente.

\section{MATERIALES Y MÉTODOS}

La localidad de Sa Pereira, departamento Las Colonias, provincia de Santa $\mathrm{Fe}$ (Argentina) conserva un importante relicto de bosque identificado en la zonificación de bosques nativos de la provincia de Santa Fe (Secretaría de Estado de Medio Ambiente y Desarrollo Sustentable, 2007) (Figura 1.A). El lugar en estudio corresponde a un sector de 100 ha de un bosque nativo que pertenece a la Escuela de Educación Técnica no 299 "Carlos Sylvestre Begnis" de Sa Pereira (Figura 1.B). La escuela se ubica en $31^{\circ} 34^{\prime} \mathrm{S}$ y $61^{\circ} 22^{\prime} \mathrm{O}$, a $54 \mathrm{~m} \mathrm{~s}$. m. y la principal vía de acceso es la Ruta Nacional $\mathrm{N}^{o}$ 19. El tipo de clima para esta zona según Köppen es subtropical húmedo (Cfa) con veranos cálidos y húmedos e inviernos frescos, donde las precipitaciones disminuyen en invierno (Castillo \& Sentis, 2001). En la zona existen amplios sectores con dominio de suelos con severas limitaciones por drenaje, Natracualfes y Natralboles (Instituto Nacional de Tecnología Agropecuaria, 1991). El sitio de estudio, si bien muestra indicios de intervención del hombre, presenta aún rasgos característicos de los bosques del Espinal. Se trata de un bosque con predominio de Prosopis alba (46\%), Vachellia caven (19\%), Celtis tala (18\%) y Geoffroea decorticans (15\%), con presencia de especies exóticas tales como Morus alba, Melia azedarach y Ligustrum lucidum (Capelino et al., 2018). 


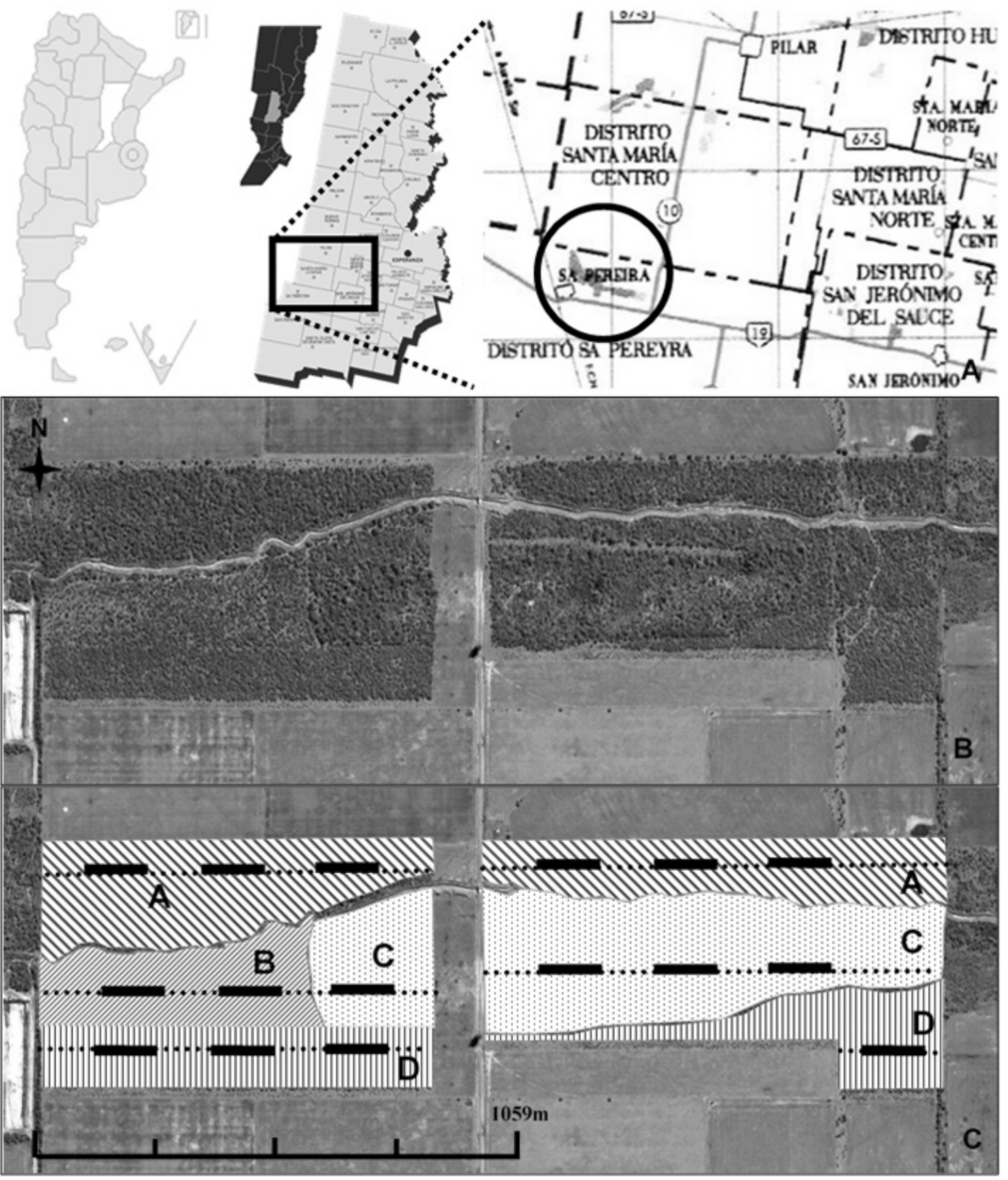

Fig. 1. A, ubicación de Sa Pereira y fragmento de la zonificación de bosques nativos (marcando con un círculo relicto de bosque en Sa Pereira). B, fotografía aérea del bosque de la Escuela de Educación Técnica n ${ }^{\circ} 299$ (31 $34^{\text {' }} \mathrm{S}$ y $61^{\circ}$ 22' O). C, estratificación propuesta para los muestreos (estratos A, B, C y D), transecto base (líneas punteadas) y líneas de muestreo cada 100 metros alternados (líneas llenas). Fuente: elaboración propia, adaptado de Secretaría de Estado de Medio Ambiente y Desarrollo Sustentable (2007) y Google Earth. 
El área basal promedio del bosque es de 12 $\mathrm{m}^{2} /$ ha con estado sanitario regular (i.e. vitalidad de copa regular o mala, fustes torcidos y enfermos) y donde el número de individuos por clase diamétrica disminuye con el aumento del diámetro (Capelino et al., 2018). Los arbustos predominantes son Senegalia praecox y Lycium boerhaviaefolium y se presentan dentro del bosque desde arbustales semi-cerrados hasta tramos desprovistos de arbustos (Capelino et al., 2018). El principal uso que se le da al bosque es el aprovechamiento del forraje por parte del ganado bovino desde marzo/abril hasta septiembre/octubre; eventualmente se extrae leña y frecuentemente se instalan apiarios para la producción de miel. Entre 1995 y 2000 se realizó una limpieza total de unas 16 a 20 has sobre el límite sur del bosque, mediante tala rasa, de las cuales una parte se mantuvo limpia para destinar a agricultura, y otra parte permaneció sin intervenciones posteriores donde se dio la regeneración natural (unidad ambiental D, Figura 1.B).

Con base en imágenes satelitales, se delimitaron cuatro segmentos de vegetación arbórea (unidades ambientales A, B, C y D, Figura 1.C). Dentro de las unidades ambientales delimitadas se procedió al muestreo en forma sistemática sobre un transecto base con orientación este-oeste (líneas en la Figura 1.C). Cada transecto se dividió en tramos de $100 \mathrm{~m}$, en un tramo se seleccionaron las dos comunidades herbáceas predominantes y se muestrearon, el siguiente tramo de $100 \mathrm{~m}$ se dejó libre, en el siguiente se volvieron a seleccionar las dos comunidades dominantes y se muestrearon y así sucesivamente. Para la identificación de las comunidades vegetales se siguió el criterio conceptual de Whittaker (1980); según éste, los límites de cada comunidad se definen con relación al área ocupada por las especies dominantes en cada una de ellas. Una vez que se identificó la comunidad herbácea más representativa del sector, se procedió al muestreo utilizando el área mínima de muestreo de $4 \times 4 \mathrm{~m}\left(16 \mathrm{~m}^{2}\right)$ definida por Pensiero et al. (2005). Una vez demarcada la superficie a muestrear, se realizó una lista con las especies presentes; al concluir la lista florística, se asignaron los valores de abundancia-cobertura de la escala de Braun-Blanquet (1979): 1: especie abundante pero de cobertura insignificante $<5 \%$; 2: abundante pero con cobertura $5-25 \%$; 3: cobertura $25-50 \%$; 4 : cobertura $50-75 \%$ y 5 : cobertura $75-100 \%$; +: poco abundante, cobertura insignificante; $r$ : muy rara con individuos solitarios; $(+)$ : presentes en la comunidad pero fuera de la unidad de muestreo. Las especies que no se identificaron a campo, fueron coleccionadas, herborizadas y posteriormente determinadas. Las determinaciones se realizaron siguiendo a Cabrera (1963, 1965a, 1965b, 1967, 1968, 1970) y Burkart (1969, 1974, 1979). Los nombres de las especies siguen el Catálogo de las Plantas Vasculares del Cono Sur (Zuloaga et al., 2019; actualizado permanentemente en http://conosur. floraargentina.edu.ar). En el Apéndice se listan todas las especies con sus respectivos autores. Para elaborar el calendario fenológico de la floración-fructificación (Apéndice), se realizaron observaciones a campo sobre los períodos de floración y fructificación, observaciones que luego se complementaron con información bibliográfica. Mediante este diseño de muestreo, se realizaron 32 parcelas (de $16 \mathrm{~m}^{2}$ ) en octubrenoviembre para el relevamiento de especies de crecimiento otoño-inverno-primaverales (OIP) y 32 en febrero-marzo para especies primaveroestivales (PE), totalizando 64 parcelas. Además, para describir el entorno de las comunidades herbáceas se discriminaron dos estratos leñosos, uno arbóreo y uno arbustivo, donde se identificaron las especies leñosas y se asignó un valor de 1 a 5 de abundancia (1: dispersos; 2: abierto; 3: semiabierto; 4: semi-cerrado; 5: cerrado).

Para caracterizar a las comunidades se indicó el número de especies (S); la proporción de gramíneas, graminoides (Cypereceae y Juncaceae) y latifoliadas; y se calcularon los índices de diversidad de Simpson (D), índice de equitatividad (E) e índice de diversidad de Shannon $(\mathrm{H})$. También se determinó la condición de la comunidad según la proporción de especies deseables, intermedias e indeseables para el ganado. La riqueza de especies (S) se define como el número de especies identificadas en un área determinada, constituyendo el concepto más antiguo y simple de la diversidad de especies (Badii et al., 2007). El problema básico en la estimación es que con frecuencia no es posible 
enumerar todas las especies en una comunidad natural y tampoco aporta información acerca de la importancia relativa de las especies (Odum, 1996; Badii et al., 2007). El índice de diversidad que propuso Simpson (1949) mide la probabilidad de que dos individuos seleccionados al azar de una comunidad sean de la misma especie. Si una especie $\mathrm{i}(\mathrm{i}=1,2$, ..., S) es representada en una comunidad por su proporción de individuos $\left(\mathrm{p}_{i}\right)$, la probabilidad de extraer al azar dos individuos pertenecientes a la misma especie, es la probabilidad conjunta $\left[\left(\mathrm{p}_{\mathrm{i}}\right)\left(\mathrm{p}_{\mathrm{i}}\right)\right.$, o $\left.\mathrm{p}_{\mathrm{i}}^{2}\right]$; y si se suman cada una de las probabilidades para todas las especies i de la comunidad, entonces el índice de diversidad de Simpson, para una muestra infinita es: $\mathrm{D}=\Sigma \mathrm{p}_{\mathrm{i}}^{2}$ (Peet, 1974; Badii et al., 2007). En este trabajo, utilizaremos el recíproco del índice de Simpson (1/D), que varía entre 1 y $\mathrm{S}$, donde $\mathrm{S}$ es el número de especies en la comunidad (Badii et al., 2007). El índice de Simpson es sensible a la abundancia de una o dos de las especies más frecuentes de la comunidad, por lo que puede ser considerado como una medida de dominancia (Badii et al., 2007). La equitatividad para el índice recíproco de Simpson se calcula como $\mathrm{E}=\mathrm{D} / \mathrm{D}_{\max }=(\Sigma$ $\mathrm{p}_{\mathrm{i}}^{2}$ ) / S (Peet, 1974). El índice de Equitatividad será igual a 1 cuando la diversidad sea la máxima posible, y próxima a cero cuando ocurre una especie fuertemente dominante (Peet, 1974). El índice de Shannon fue calculado mediante la siguiente fórmula $\mathrm{H}=-\Sigma \mathrm{p}_{\mathrm{i}} \ln \mathrm{p}_{\mathrm{i}}$; donde $\mathrm{p}_{\mathrm{i}}$ es la proporción del número total de individuos que constituyen la iésima especie (Peet, 1974; Odum, 1996; Badii et al., 2007). El índice de Shannon es igual a cero si sólo hay una especie en la muestra y es máximo si todas las especies están representadas por el mismo número de individuos (Peet, 1974; Odum, 1996; Badii et al., 2007). En la práctica las comunidades biológicas parecen no exceder el valor de diversidad de Shannon $(\mathrm{H})$ de 5 (Badii et al., 2007).

Se determinó la importancia relativa de las comunidades en cada unidad ambiental, midiendo el inicio y el fin de cada comunidad herbácea siguiendo las líneas de la transecta base (Figura 1.C) y asignando un valor de 1 a 3 según la extensión en ancho (1: menor a $20 \mathrm{~m} ; 2: 20-50 \mathrm{~m}$ y 3: mayor a $50 \mathrm{~m}$ ) (Pensiero \& D'Angelo, 2006).
La condición del pastizal (o las comunidades) se define, desde un punto de vista productivo, como el estado de salud en que se encuentra el mismo; es decir, es la cuantificación del deterioro de la aptitud forrajera de un pastizal con relación a la vegetación más productiva para el sitio (Bissio, 1984; Díaz, 1992). La condición depende de la relación porcentual entre las especies consideradas deseables, intermedias e indeseables (Dyksterhuis, 1949; Bissio, 1984; Díaz, 1992; Pensiero \& D'Angelo, 2006). Especies "deseables" son aquellas que, por ser las preferidas por el ganado (mayor palatabilidad, mayor digestibilidad), son consumidas en primer término y con mayor intensidad (Bissio, 1984; Díaz, 2007). Las especies "intermedias" son aquellas que, si bien no son las más palatables, el animal consume sin inconvenientes, y por lo general el incremento de la frecuencia de especies intermedias va acompañado por una disminución de la frecuencia de especies deseables (Bissio, 1984; Pensiero \& D'Angelo, 2006; Díaz, 2007). Las especies "indeseables" son aquellas plantas no consumidas por el ganado (especies con espinas, tóxicas, con sabor amargo, con baja digestibilidad) y que en general presentan un comportamiento invasor; estas especies además se caracterizan por ocupar rápidamente los espacios que dejan las especies intermedias y deseables (Díaz, 1992; Pensiero \& D’Angelo, 2006; Marino, 2008).

\section{RESULTADOS}

En el estrato herbáceo, las comunidades identificadas en los muestreos OIP y PE se encontraron dispuestas espacialmente en forma de mosaico o parches y fueron tres: flechillar, pradera húmeda y gramillar, distribuidas en ocho variantes: flechillar, flechillar degradado, flechillar húmedo, pradera húmeda, pradera húmeda degradada, gramillar, gramillar húmedo y gramillar salino. Las características de las comunidades, las variaciones según condición, el ambiente específico y las principales especies presentes (ver Apéndice con la lista de especies para cada comunidad) fueron: 
I-i) Flechillar: esta comunidad ocurrió bajo la influencia de la copa de los árboles (cobertura arbórea semi-abierta a cerrada) y se ubicó en los sectores más altos del terreno. Está dominada por flechillas, entre las que se destacaron flechilla mansa (Nassella hyalina) y flechilla brava (Nassella neesiana), además de falsa flechilla (Piptochaetium stipoides), cola de zorro (Setaria fiebrigii), cebadilla criolla (Bromus catharticus), entre otras. En los muestreos PE dominaron falsa flechilla (Piptochaetium stipoides) y barabal (Setaria parviflora).

I-ii) Flechillar degradado: presentó una pobre presencia de flechillas y cebadilla, y un aumento de la frecuencia de especies de hojas anchas, entre las que se destacaron escoba dura (Sida rhombifolia) y eupatorio (Austroeupatorium, Chromolaena o Urolepis); además de gramilla rastrera (Cynodon dactylon) y pasto serrucho (Melica macra). En los muestreos PE estas especies indeseables aumentaron en detrimento de flechillas y barabal.

I-iii) Flechillar húmedo: presentó un aumento de aquellas especies que crecen en situaciones o suelos con más humedad, como ciperáceas y juncáceas, destacándose los géneros Cyperus, Carex y Juncus. Se distinguió, además, la presencia de oreja de ratón (Dichondra microcalyx), alpistillo (Phalaris angusta) y yuyito de la pared (Parietaria debilis).

II-i) Pradera húmeda: esta comunidad ocurrió en abras del bosque (cobertura arbórea abierta a semi cerrada), en sitios ligeramente bajos, deprimidos, en suelos húmedos y con escasa o nula presencia de sales, con predominio de especies del género Paspalum y los géneros Cyperus, Carex y Juncus. En los muestreos OIP se distinguió, además, una importante presencia de oreja de ratón (Dichondra microcalyx) y alpistillo (Phalaris angusta).

II-ii) Pradera húmeda degradada: en esta comunidad, a diferencia de la pradera húmeda, aumentó la frecuencia de la gramilla rastrera y latifoliadas (Sida rhombifolia, Hyptis sp., Austroeupatorium sp., Chromolaena sp., Urolepis $s p$., Senecio sp., entre otros) en detrimento del pasto miel (Paspalum dilatatum) y pasto horqueta (Paspalum notatum).

III-i) Gramillar: ocurrió en abras del bosque (cobertura arbórea abierta y árboles dispersos), en sitios ligeramente altos de suelos con menor capacidad para retener humedad y con presencia variable de sales. Si bien se encontraron diversas especies en esta pradera, la dominante fue la gramilla rastrera (Cynodon dactylon).

III-ii) Gramillar húmedo: además de gramilla hubo una importante presencia de especies ciperáceas y juncáceas destacándose los géneros Cyperus, Carex y Juncus. También se presentaron especies como Dichondra microcalyx y Setaria geminata.

III-iii) Gramillar salino: aquí predominaron los géneros Cynodon, Chloris, Sporobolus y Eleusine, también ocurrieron especies halófilas como cola de gama (Heliotropium curassavicum).

Hay que notar aquí, que registramos otra comunidad que llamamos de canutillar y que consideramos secundaria debido a su baja proporción y escasa extensión. Por esta razón no se incluyó en los censos del área mínima de muestreo mediante Braun-Blanquet. Sin embargo, sí la incluimos en la determinación de la importancia relativa de las comunidades (Tabla 1).

Considerando toda la vegetación relevada en el bosque de Sa Pereira, se registraron 45 familias y 156 especies (S) (ver Apéndice), siendo las familias más representadas Poaceae (38 especies) y Asteraceae (25 especies). En promedio se registraron 20,8 y 18,1 especies por parcela para los muestreos OIP y PE respectivamente.

Tabla 1. Superficie, densidad de árboles, proporción de especies arbóreas (\%) y proporción de comunidades herbáceas $(\%)$ por unidades ambientales.

\begin{tabular}{|c|c|c|c|c|c|}
\hline \multicolumn{2}{|c|}{ Unidad ambiental } & A & B & $\mathrm{C}$ & $\mathrm{D}$ \\
\hline \multicolumn{2}{|c|}{ Superficie (ha) * } & 28 & 12 & 38 & 22 \\
\hline \multicolumn{2}{|c|}{ Árboles/ha * } & 385 & 360 & 310 & 495 \\
\hline \multirow{5}{*}{$\begin{array}{l}\text { Frecuencia de } \\
\text { árboles }(\%) *\end{array}$} & Prosopis alba & 51 & 29 & 29 & 58 \\
\hline & Vachellia caven & 7 & 32 & 2 & 41 \\
\hline & Celtis tala & 32 & 10 & 23 & 0.5 \\
\hline & Geoffroea decorticans & 9 & 25 & 46 & 0.5 \\
\hline & Otras & 1 & 4 & 0 & 0 \\
\hline \multirow{4}{*}{$\begin{array}{c}\text { Frecuencia de } \\
\text { comunidades } \\
(\%)\end{array}$} & Flechillares & 40 & 20 & 27 & 4 \\
\hline & Praderas húmedas & 19 & 28 & 19 & 50 \\
\hline & Gramillares & 38 & 52 & 53 & 46 \\
\hline & Secundarias (Canutillar) & 3 & 0 & 2 & 0 \\
\hline
\end{tabular}

* Capelino et al., 2018 
Tabla 2. Parámetros para describir los conjuntos de comunidades en los dos muestreos estacionales.

\begin{tabular}{|c|c|c|c|c|c|c|c|}
\hline \multirow{2}{*}{\multicolumn{2}{|c|}{$\begin{array}{l}\text { Época de muestreo } \\
\text { Comunidad }\end{array}$}} & \multicolumn{3}{|c|}{ Otoño-inverno-primaveral } & \multicolumn{3}{|c|}{ Primavero-estival } \\
\hline & & Flechillares & P.H. & Gramillares & Flechillares & P.H. & Gramillares \\
\hline \multicolumn{2}{|c|}{$\mathrm{n}^{\mathrm{o}}$ de muestreos } & 18 & 6 & 8 & 9 & 14 & 9 \\
\hline \multicolumn{2}{|c|}{$\mathrm{n}^{\mathrm{o}}$ de especies medio (S) } & 22,6 & 24,3 & 13,9 & 19,1 & 18,6 & 16,2 \\
\hline \multicolumn{2}{|c|}{ Índice de Simpson medio (1/D) } & 7,68 & 8,43 & 3,26 & 9 & 8,75 & 7,3 \\
\hline \multicolumn{2}{|c|}{ Índice de equitatividad medio (E) } & 0,34 & 0,35 & 0,23 & 0,48 & 0,46 & 0,46 \\
\hline \multicolumn{2}{|c|}{ Índice de Shannon medio $(\mathrm{H})$} & 2,28 & 2,37 & 1,47 & 2,34 & 2,32 & 2,2 \\
\hline \multirow{3}{*}{$\begin{array}{l}\text { Frecuencia de } \\
\text { especies }(\%)\end{array}$} & Gramíneas & 50,4 & 38,7 & 75,9 & 44,6 & 23,2 & 67,8 \\
\hline & Graminoides & 21,1 & 31,3 & 19,5 & 27,4 & 33,1 & 12,8 \\
\hline & Latifoliadas & 28,8 & 29,8 & 4,7 & 28,1 & 44,7 & 19,4 \\
\hline \multirow{3}{*}{$\begin{array}{l}\text { Frecuencia de } \\
\text { especies (\%) }\end{array}$} & Deseables & 37,5 & 31,9 & 72,8 & 26,3 & 7,5 & 57,8 \\
\hline & Intermedias & 41,4 & 39,6 & 23,3 & 56,6 & 67,8 & 26,5 \\
\hline & Indeseables & 21,1 & 28,5 & 3,9 & 17,2 & 24,7 & 15,7 \\
\hline \multicolumn{2}{|c|}{ Condición media del pastizal } & $\mathrm{R}$ & $\mathrm{R}$ & $\mathrm{E}$ & $\mathrm{R}$ & M & $\mathrm{B}$ \\
\hline \multicolumn{2}{|c|}{ Cobertura media de árboles } & 3 & 3 & 2 & 3 & 3 & 2 \\
\hline \multicolumn{2}{|c|}{ Cobertura media de arbustos } & 1 & 1 & 2 & 2 & 1 & 2 \\
\hline
\end{tabular}

Flechillares: incluye las comunidades flechillar, fechillar degradado y flechillar húmedo. P.H., pradera húmeda: incluye las comunidades pradera húmeda y pradera húmeda degradada. Gramillares: incluye las comunidades gramillar, gramillar húmedo y gramillar salino. Condición del pastizal (según la proporción de especies deseables, intermedias e indeseables): E: excelente, B: buena, R: regular, M: mala. Cobertura de árboles y de arbustos: 1: aislados; 2: abierto; 3: semi-abierto; 4: semi-cerrado; 5: cerrado.

Las praderas húmedas mostraron mayor número de especies en los relevamientos OIP, seguidas por los flechillares y gramillares (Tabla 2). En los muestreos PE los flechillares mostraron el mayor número de especies, seguidos por las praderas húmedas y los gramillares (Tabla 2). En los muestreos OIP, se registraron en promedio 55 $\%$ de gramíneas, $22 \%$ de graminoides y $23 \%$ de latifoliadas. En los muestreos $\mathrm{PE}$ en promedio la proporción de latifoliadas se incrementó en detrimento de las gramíneas, manteniéndose muy similares las proporciones de graminoides. En la Tabla 2 se presentan las particularidades para cada grupo de comunidades.

En los muestreos OIP y PE se obtuvo un índice de diversidad de Simpson (interpretado como su recíproco, 1/D) promedio de 6,72 y 8,42 respectivamente. En los muestreos OIP las praderas húmedas mostraron el mayor índice de diversidad de Simpson, seguido por los flechillares y los gramillares. En los muestreos PE los flechillares fueron más diversos según el índice de Simpson seguido por las praderas húmedas y los gramillares (Tabla 2). Los valores de equitatividad del índice recíproco de Simpson (E) medio para los muestreos OIP fueron, en general, menores que los PE para todos los grupos de comunidades (Tabla 2). Los menores valores de equitatividad fueron 0,11 y 0,22 en los muestreos OIP y PE respectivamente, correspondiéndose en ambos casos con un gramillar donde Cynodon dactylon adquirió un alto valor de abundancia-cobertura. Las comunidades con mayor equitatividad (casos puntuales dentro de los grupos de comunidades) fueron un flechillar húmedo $(0,49)$ y una pradera húmeda $(0,66)$ para ambos muestreos respectivamente. El índice de Shannon $(\mathrm{H})$ varió entre 1,04 y 2,86 para los muestreos OIP y entre 1,76 y 2,84 en los muestreos PE. Las praderas húmedas presentaron los mayores índices de Shannon seguidas por los flechillares y gramillares para los muestreos OIP (Tabla 2). 
En los muestreos OIP se registraron en promedio $45 \%$ de especies deseables, $37 \%$ de especies intermedias y $18 \%$ de indeseables. Para todas las comunidades en los muestreos PE, disminuyó la proporción de especies deseables $(27 \%)$ en favor de la intermedias (53\%), manteniéndose las indeseables con poca variación (20\%) respecto de los muestreos OIP. En la Tabla 2 pueden observarse las particularidades para cada grupo de comunidades y la condición de la comunidad según la proporción de especies deseables, intermedias e indeseables.

\section{DISCUSIÓN}

Las comunidades herbáceas se encontraron dispuestas en forma de parches o mosaicos, lo cual puede asociarse a variaciones en el micro relieve o a características específicas del suelo que influyen en la distribución de las especies y por lo tanto en las extensiones y distribución de las comunidades. Patrones de distribución semejantes (en mosaico) han sido observados en bosques de Schinopsis balansae del Chaco Húmedo (Marino \& Pensiero, 2003). En general, las diferentes comunidades del pastizal se corresponden con ubicaciones específicas en el paisaje y con rangos característicos de propiedades de los suelos (Pucheta et al., 1998; Marino \& Pensiero, 2003; Batista et al., 2005). Por otra parte, se observó que estas comunidades herbáceas estuvieron asociadas a variaciones en la abundancia y cobertura del estrato arbóreo, donde los flechillares estuvieron asociados con mayor cobertura arbórea, seguidos por praderas húmedas y gramillares (Tabla 1 y 2); esto muestra que la presencia de árboles puede afectar la composición florística, la fenología, la productividad y la distribución de la biomasa de los estratos inferiores (Sabattini et al., 2002; Díaz, 2007).

La vegetación está formada por un conjunto relativamente reducido de combinaciones de especies de la flora local (comunidades vegetales) que se repiten en diferentes sitios dentro del paisaje formando un diseño característico (Batista et al., 2005). El predominio de flechilla mansa (Nassella hyalina), flechilla brava (Nassella neesiana), falsa flechilla (Piptochaetium stipoides), pasto miel (Paspalum dilatatum), pasto horqueta (Paspalum notatum) y gramilla rastrera (Cynodon dactylon), entre otras, demuestran la importancia del flechillar, pradera húmeda y gramillar como comunidades dominantes. Estas comunidades son equivalentes (en cuanto a especies herbáceas dominantes) a las halladas por otros autores en distintas localidades como San Javier, San Cristóbal y Esperanza (Marino \& Pensiero, 2003; Pensiero et al., 2005; Pensiero \& D'Angelo, 2006; Bugnon \& Gutiérrez 2006).

Del total de 156 especies determinadas, la mayor cantidad correspondió a las familias Poaceae y Asteraceae. Este número de especies se encuentra entre las 263 especies citadas por Exner et al. (2004) en la Reserva de la Escuela Granja de Esperanza y las 95 especies que distinguieron Lewis et al. (2006) en la zona de Coronda. La diferencia en la cantidad de especies encontradas podría ser explicada por variables edáficas, historia del relicto, manejo de los pastizales, efecto del estrato arbóreo, metodología de muestreo, entre otras. Los valores encontrados en este relicto de bosque son interesantes ya que la diversidad florística constituye una característica importante de los ecosistemas, tanto por sus implicancias para la conservación de especies como por su rol en el funcionamiento de ecosistemas, incrementando la eficiencia de uso de los recursos y contribuyendo a estabilizar el funcionamiento de los ecosistemas frente a factores de estrés o disturbio (Loydi \& Distel, 2010).

Las comunidades vegetales con mayor número de especies fueron los flechillares y las praderas húmedas. Estos valores de riqueza específica se aproximan a los mencionados en trabajos equivalentes realizados en San Cristóbal y San Javier. Pensiero et al. (2005) señalan para un establecimiento en el departamento San Cristóbal (Santa $\mathrm{Fe}$ ) que el número de especies para flechillares estuvo entre 17 y 47 especies, para gramillares entre 16 y 35 , y para praderas húmedas 21 especies. También mencionan para un establecimiento en el departamento San Javier (Santa $\mathrm{Fe}$ ) que la riqueza de las comunidades fue: flechillar 25 especies, pradera húmeda $15 \mathrm{y}$ gramillar 15 especies. 
Las comunidades menos diversas según el índice de Simpson (interpretado como su recíproco, 1/D) fueron los gramillares, donde predominó Cynodon dactylon. El índice de Simpson mide tanto la dominancia como la riqueza, y en comparación con el Índice de Shannon, hace más énfasis en la riqueza de especies que en la abundancial (Peet, 1974). Cuanto mayor sea este índice habrá mayor diversidad y la probabilidad de que dos individuos pertenezcan a la misma especie será menor. Se observa que hay mayor diversidad cuantas más especies tiene la comunidad, esto se da claramente para flechillares y praderas húmedas.

Las comunidades de praderas húmedas $\mathrm{y}$ flechillares presentaron los mayores valores de equitatividad del recíproco de Simpson, lo que evidencia la mayor uniformidad en la distribución de individuos entre las especies dominantes de estas comunidades. El menor valor de equitatividad correspondió a los gramillares, atribuido principalmente a la presencia de Cynodon dactylon, especie que mostró alto valor de abundancia-cobertura. Los valores de equitatividad promedio por grupo de comunidades para los muestreos PE muestran valores similares $(0,46-0,48)$ lo que sugiere que las especies presentan una heterogeneidad equivalente, por lo que habrá especies que compartan abundancias similares y otras especies que no. Dentro de cada grupo de comunidades encontramos dispersión de datos, por ejemplo, en los gramillares PE encontramos valores de equitatividad de 0,2 y 0,59 para un gramillar en condición regular y un gramilllar húmedo en condición buena respectivamente.

Según el índice de Shannon praderas húmedas y flechillares fueron en general más diversas que gramillares para ambos muestreos. Es esperable que el disturbio producido por el ganado, que no fue medido en este trabajo, sea uno de los factores responsables de diferencias en la abundancia y diversidad. Los animales en pastoreo desarrollan un hábito de consumo selectivo, esto significa que los vacunos perciben la heterogeneidad florística $\mathrm{y}$ de comunidades ejerciendo un uso diferencial muy marcado, conllevando consecuentemente al sobreuso de los ambientes preferidos y a la subutilización de las áreas rechazadas (Díaz, 1992;
Cauhépé \& Hidalgo, 2005). Sabattini et al. (2008) citan que, en pastizales incorporados recientemente al pastoreo, la condición del pastizal natural está relacionada con la diversidad. Además mencionan que el índice de diversidad de Shannon aumenta a medida que mejora la condición del pastizal desde mala a buena; pero disminuye al alcanzar la condición excelente, observando índices de diversidad de 0,66 a 2,58; en el presente estudio variaron entre 1,04 y 2,86 . Nai-Bregaglio et al. (2002) hallaron, en pastizales de las sierras de Córdoba, valores de diversidad de especies mayores en los sitios pastoreados $(\mathrm{H}$ $=3,60 \pm 0,19)$ respecto de los sitios excluidos al ganado $(\mathrm{H}=1,28 \pm 0,11)$; por su parte Altesor et al. (2005) y Rush \& Oesterheld (1997) arriban a similares conclusiones en pastizales en Uruguay $\mathrm{y}$ en Ayacucho (provincia de Buenos Aires) respectivamente.

La información generada en este trabajo, como las comunidades identificadas y caracterizadas, su importancia relativa y distribución, y la condición de las comunidades es un gran aporte a la descripción cuantitativa y cualitativa de la vegetación herbácea en el Espinal Santafesino, y en especial al relicto de Bosque de la Escuela de Educación Técnica $n^{\circ}$ 299. Esta información podría utilizarse para estimar la producción de las comunidades en particular y del pastizal del predio en general. Esto permitiría tener una idea más concreta de la cantidad de animales que soportaría este ecosistema para realizar un aprovechamiento racional del mismo. Se propone la instalación de jaulas de exclusión sobre las comunidades más representativas para determinar la productividad de materia seca in situ, al menos durante tres años para obtener así valores de productividad promedio representativos que se ajustarán a las condiciones locales. Así mismo sería conveniente continuar con los relevamientos florísticos, aprovechando especialmente las parcelas previas a pastorear, lo que permitirá una caracterización más completa y acabada sobre la flora y vegetación en general y sobre las comunidades en particular. Por todo esto, conocer el estado actual de un ecosistema debería ser el insumo inicial para la realización de cualquier práctica relativa a la producción o conservación de los mismos. 


\section{BIBLIOGRAFÍA}

Altesor, A.; M. Oesterheld, E. Leoni, F. Lezama \& C. Rodríguez. 2005. Effect of grazing on community structure and productivity of a Uruguayan grassland. Plant Ecology 179(1): 83-91.

Atlas de los Bosques Nativos Argentinos. 2003. Proyecto bosques nativos y áreas protegidas BIRF 4085-AR. Dirección de Bosques, Secretaría de Ambiente y Desarrollo Sustentable. 245 pp.

Badii, M. H.; J. Landeros \& E. Cerna. 2007. Patrones de asociación de especies y sustentabilidad. Daena: International Journal of Good Conscience 3(1): 632-660.

Batista, W. B.; M. A. Taboada, R. S. Lavado, S. B. Perelman \& R. J. C. León. 2005. Asociación entre comunidades vegetales y suelos en el pastizal de la Pampa Deprimida, en Oesterheld, M.; M. R. Aguiar, C. M. Ghersa and J. M. Paruelo (eds.), La heterogeneidad de la vegetación de los agroecosistemas, pp. 113-119. Editorial Facultad de Agronomía UBA, Buenos Aires, Argentina.

Bissio, J. C. 1984. Manejo de pastizales -Conceptos y breve introducción a las técnicas utilizadas-. Publicación Miscelánea $\mathrm{n}^{\circ}$ 2. INTA EEA Reconquista. Santa Fe, Argentina. 22 pp.

Braun-Blanquet, J. 1979. Fitosociología -bases para el estudio de las comunidades vegetales-. Madrid, España. Blume Ediciones. 820 pp.

Bugnon, M. \& H. Gutiérrez. 2006. Censado de pastizales -para la determinación de la condición y la estimación de la productividad-. Apunte de cátedra Nodo I-Botánica Sistemática. Universidad Nacional del Litoral-Facultad de Ciencias Agrarias (UNL-FCA) Santa Fe, Argentina. 4 pp.

Burkart, A. 1969, 1974, 1979. Flora Ilustrada de Entre Ríos. Colección Científica I.N.T.A. Tomo VI. Vols. II, IV \& V, Bs. As.

Cabrera, A. L. 1963, 1965a, 1965b, 1967, 1968, 1970. Flora de la Provincia de Buenos Aires. Colección Científica I.N.T.A. Tomo IV, 6 Vols, Bs. As.

Cabrera, A. L. 1976. Regiones fitogeográficas argentinas, en W. F. Kugler (ed.), Enciclopedia Argentina de Agricultura y Jardinería. Tomo 2. 2da edición.Acme, Buenos Aires, Argentina. Fascículo 1. pp. 1-85.

Capelino, P.; A. Bender \& P. Hernández. 2018. Evaluación de la vegetación leñosa de un algarrobal del Espinal de la provincia de Santa Fe (Argentina). Quebracho Vol.26 (1,2): 51-59

Castillo, F. E. \& F. C. Sentis. 2001. Agrometeorología. Madrid, España. Mundi-Prensa. 517 pp.
Cauhépé, M. \& L. Hidalgo. 2005. La Pampa Inundable: el uso ganadero como base de la sustentabilidad social, económica y ambiental, en Oesterheld M.; M. Aguiar, C. Ghersa, J. Paruelo (eds.), La heterogeneidad de la vegetación de los agroecosistemas, pp. 403-414. Buenos Aires, Argentina. Editorial Facultad de Agronomía (UBA).

Deregibus, V. A. 1988. Importancia de los pastizales naturales en la República Argentina: situación presente y futura. Revista Argentina de Producción Animal 8(1): 67-78.

Díaz, R. 1992. Evaluación de los recursos forrajeros del Chaco árido, en Karlini UO, R Coirini (eds.), Sistemas agroforestales para pequeños productores de zonas áridas, pp. 18-23. Córdoba, Argentina. Coordinación Editorial Irastorza.

Díaz, R. 2007. Utilización de pastizales naturales. Argentina. Editorial Brujas. 456 pp.

Dyksterhuis, E. J. 1949. Condition and management of rangeland based on quantitative ecology. Journal of Range Management 2: 104-115.

Escobar, J. M. 2008. Los Pastizales Naturales de la Patagonia, en INTA (Instituto Nacional de Tecnología Agropecuaria, AR) (ed.), Manejo de pastizales, evaluación predial, pp. 3-7. Trelew, Argentina.

Exner, E.; C. D'Angelo \& J. Pensiero. 2004. Vegetación y flora de la Reserva Universitaria de la Escuela Granja de Esperanza (Santa Fe, Argentina). Revista FAVE - Ciencias Agrarias 3(1-2): 53-76.

Instituto Nacional de Tecnología Agropecuaria (INTA, AR) 1991. Carta de suelos de la República Argentina. EsperanzaPilar. Santa Fe, Argentina. INTA Rafaela. 135 pp.

Karlin, U. \& J. Bernasconi. 2005. Tecnologías tradicionales. PIARFON (Proyectos de Investigación Aplicada a los Recursos Forestales Nativos). Proyecto Bosques Nativos y Áreas Protegidas BIRF 4085 - AR Región del Monte y del Espinal. Secretaría de Ambiente y Desarrollo Sustentable de la Nación. Universidad Nacional de Entre Ríos. Universidad Nacional del Litoral. Universidad Nacional de Córdoba. 14 pp.

Lewis, J. P.; E. P. Pire, I. M. Barberis \& D. E. Prado. 2006. Los bosques del espinal periestépico en las proximidades de la localidad de Coronda, Provincia de Santa Fe (Argentina). Revista de Investigaciones de la Facultad de Ciencias Agrarias (Universidad Nacional de Rosario) 10: 13-26.

Loydi, A. \& R. Distel, 2010. Diversidad florística bajo diferentes intensidades de pastoreo por grandes herbívoros en pastizales serranos del Sistema de Ventania, Buenos Aires. Ecología Austral 20: 281-291. Asociación Argentina de Ecología. 
Marino, G. 2008. Buenas prácticas ganaderas para conservar la vida silvestre de las pampas: una guía para optimizar la producción y conservar la biodiversidad de los pastizales de la Bahía Samborombón y la Cuenca del Río Salado. Aves Argentinas/Asociación Ornitológica del Plata, Buenos Aires. Coeditado con la Fundación Vida Silvestre Argentina y BirdLife International. 107 pp.

Marino, G. \& J. Pensiero. 2003. Heterogeneidad florística y estructural de los bosques de Schinopsis balansae (Anacardiaceae) en el sur del Chaco Húmedo. Darwiniana 41(1-4): 17-28.

Matteucci, S. 2012. Ecorregión Espinal, en Morello, J.; S. D. Matteucci, A. Rodriguez, M. Silva (eds.), Ecorregiones y Complejos Ecosistémicos Argentinos, Primera Edición, Capítulo 11, pp. 349-390. Imprenta Orientación Gráfica Editora S.R.L.

Nai-Bregaglio, M.; C. Pucheta \& M. Cabido. 2002. El efecto del pastoreo sobre la diversidad florística y estructural en pastizales de montaña del centro de Argentina. Revista Chilena de Historia Natural 75: 613-623.

Odum, E. P. 1996. Ecología (3 ${ }^{a}$ Edición). Nueva editorial Interamericana. Prensa Técnica S.A. D.F. México. 639 pp.

Peet, R. K. 1974. The Measurement of Species Diversity. Annual Review of Ecology and Systematics 5: 285-307.

Pensiero, J. \& C. D’Angelo. 2006. Componente pastizales naturales, en Mesa Agroforestal Santafesina (ed.), Bosques para siempre -las prácticas para un manejo sustentable de los bosques santafesinos-, pp. 25-46. Santa Fe, Argentina. Imprenta Daphereikon.

Pensiero, J.; G. Marino, C. D'Angelo \& C. Dimundo. 2005. Informe de la actividad pastizales de la Provincia de Santa Fe. Santa Fe, Argentina. 68 pp.

Proyectos de Investigación Aplicada a los Recursos Forestales Nativos (PIARFON), 2005. Estudio Forestal. Proyecto Bosques Nativos y Áreas Protegidas BIRF 4085 - AR Región del Monte y del Espinal. Secretaría de Ambiente y Desarrollo Sustentable de la Nación. Universidad Nacional de Entre Ríos. Universidad Nacional del Litoral. Universidad Nacional de Córdoba. 44 pp.

Pucheta, E.; F. Vendramini, M. Cabido \& S, Díaz. 1998. Estructura y funcionamiento de un pastizal de montaña bajo pastoreo y su respuesta luego de su exclusión. Revista de la Facultad de Agronomía, La Plata 103(1): 77-92.

Rush, G. M. \& M. Oesterheld. 1997. Relationship between productivity, and species and funcional group diversity in grazed and non-grazed Pampas grassland. Oikos 78: 519-526.

Sabattini, R.; S. Sione S. Ledesma, B. Muracciole, F. Cottani \& C. Fortini. 2008. Análisis de la diversidad florística y de los tipos productivos del pastizal natural en un monte nativo bajo pastoreo rotativo (Entre Ríos, Argentina). RCA. Revista de Investigaciones Agropecuarias 12(1): 5-13.

Sabattini, R. A.; N. Muzzachiodi \& A.F. Dorsch. 2002. Manual de prácticas de manejo del monte nativo. Entre Ríos, Argentina. 56 pp.

Simpson, E. H. 1949. Measurement of diversity. Nature 163: 688.

Secretaría de Estado de Medio Ambiente y Desarrollo Sustentable (SMADES, AR). 2007. Zonificación del bosque nativo. Sobre la parcela rural de la provincia de Santa $\mathrm{Fe}$, por medio de imágenes satelitales. Santa Fe, Argentina. Acosta Hnos. S.H. 48 pp.

Whittaker, R. H. 1980. Dominance Types, en Whittaker, R. H. (ed.), Classification of Plant Comunities, pp. 65-79. Junk, The Hague.

Zuloaga, F. O.; M. J. Belgrano \& C. A. Zanotti. 2019. Actualización del catálogo de plantas vasculares del Cono Sur. Darwiniana, nueva serie 7(2): 208-278. 
Apéndice. Lista florística de familias y especies por comunidades herbáceas. Se indica la forma de vida, origen y época de floración y fructificación.

\begin{tabular}{|c|c|c|c|c|c|c|c|c|c|c|}
\hline & & & & & & Comu & idades & sus va & riantes & \\
\hline pecie & $\begin{array}{l}\text { Forma } \\
\text { de vida }\end{array}$ & Origen & /fructif. & Flech. & $\begin{array}{c}\text { Flech. } \\
\text { Deg. }\end{array}$ & $\begin{array}{c}\text { Flech. } \\
\text { H. }\end{array}$ & P.H. & $\begin{array}{l}\text { P.H. } \\
\text { Deg. }\end{array}$ & Gram. & Gram. \\
\hline
\end{tabular}

\section{DICOTILEDONEAS}

\section{ACANTHACEAE}

Dicliptera squarrosa Nees

Hi p N PVO

\section{AMARANTHACEAE}

Amaranthus muricatus (Moq.)

Hieron.

Hi a N $\quad$ VO

Gomphrena perennis L.

Hi p N VO

Iressine diffusa Humb. \& Bonpl. ex Willd.

Hi p N VO

Pfaffia glomerata (Spreng.)

Pedersen

Hi p N PV

\section{APIACEAE}

Bowlesia incana Ruiz \& Pav.

Hi a N PVO

Eryngium coronatum Hook. \&

Arn.

Hi p N

Eryngium echinatum Urb.

Eryngium paniculatum Cav. \& Dombey ex F. Delaroche

Hi p N PV

Hi p N PV

\section{APOCYNACEAE}

Araujia brachystephana (Griseb.) Fontella \& Goyder

En $\mathrm{p} \quad \mathrm{N}$

Araujia odorata (Hook. \& Arn.)

Fontella \& Goyder

En $\mathrm{p} \quad \mathrm{N}$

VO

ASTERACEAE

Anthemis cotula $\mathrm{L}$.

Artemissia annua $\mathrm{L}$.

Hi a E PV

Austroeupatorium inulifolium (Kunth) R.M. King \& H. Rob.

Hi a E PV

Hi a N VO

Baccharis articulata (Lam.) Pers. ar $\quad$ N $\quad$ PV

Baccharis coridifolia DC.

Hi p N $\quad \mathrm{V}$

Baccharis glutinosa Pers.

Hi p N $\quad$ VO

Bidens subalternans DC.

Hi a N P

Carduus nutans L.

Hi a-b E

Chaptalia nutans (L.) Pol.

Hi p N $\quad \mathrm{P}$




\section{P. A. CAPELINO \& A. G. BENDER. Vegetación herbácea Espinal}

Apéndice. (Continuación). Lista florística de familias y especies por comunidades herbáceas. Se indica la forma de vida, origen y época de floración y fructificación.

\begin{tabular}{|c|c|c|c|c|c|c|c|c|c|c|c|}
\hline \multirow[b]{2}{*}{ Familia y especie } & \multirow{2}{*}{$\begin{array}{l}\text { Forma } \\
\text { de vida }\end{array}$} & \multirow[b]{2}{*}{ Origen } & \multirow{2}{*}{$\begin{array}{l}\text { Floración } \\
\text { /fructif. }\end{array}$} & \multicolumn{8}{|c|}{ Comunidades y sus variantes } \\
\hline & & & & Flech. & $\begin{array}{l}\text { Flech. } \\
\text { Deg. }\end{array}$ & $\begin{array}{l}\text { Flech. } \\
\text { H. }\end{array}$ & P.H. & $\begin{array}{l}\text { P.H. } \\
\text { Deg. }\end{array}$ & Gram. & $\begin{array}{l}\text { Gram. } \\
\text { H. }\end{array}$ & $\begin{array}{l}\text { Gram. } \\
\text { Sal. }\end{array}$ \\
\hline $\begin{array}{l}\text { Chromolaena christieana (Baker) } \\
\text { R.M. King \& H. Rob. }\end{array}$ & Hi a & $\mathrm{N}$ & VO & $*$ & * & * & $*$ & * & $*$ & $*$ & $*$ \\
\hline Cirsium vulgare (Savi) Ten. & $\mathrm{Hi} \mathrm{a}$ & $\mathrm{E}$ & PV & & & * & $*$ & $*$ & & & \\
\hline Conyza bonariensis (L.) Cronquist & Hi a & $\mathrm{N}$ & $\mathrm{VO}$ & * & * & $*$ & $*$ & * & $*$ & $*$ & $*$ \\
\hline $\begin{array}{l}\text { Cotula australis (Sieber ex } \\
\text { Spreng.) Hook. f. }\end{array}$ & Hi a & $\mathrm{E}$ & IP & & & & $*$ & & & & \\
\hline $\begin{array}{l}\text { Gamochaeta americana (Mill.) } \\
\text { Wedd. }\end{array}$ & Hi b-p & $\mathrm{N}$ & $P$ & * & $*$ & $*$ & $*$ & $*$ & $*$ & & \\
\hline $\begin{array}{l}\text { Hymenoxis anthemoides (Juss.) } \\
\text { Cass. }\end{array}$ & Hi $\mathrm{p}$ & $\mathrm{N}$ & $P$ & & & & & & & * & \\
\hline $\begin{array}{l}\text { Hypochaeris microcephala (Sch. } \\
\text { Bip.) Cabrera }\end{array}$ & Hi $p$ & $\mathrm{~N}$ & PV & $*$ & $*$ & * & $*$ & $*$ & $*$ & $*$ & \\
\hline $\begin{array}{l}\text { Lessingianthus rubricaulis } \\
\text { (Bonpl.) H. Rob. }\end{array}$ & Hi $p$ & $\mathrm{~N}$ & PV & * & $*$ & & $*$ & & & & \\
\hline Picrosia longifolia D. Don & Hi $\mathrm{p}$ & $\mathrm{N}$ & $\mathrm{V}$ & $*$ & & & $*$ & & & & \\
\hline Pterocaulon virgatum (L.) DC. & Hi $\mathrm{p}$ & $\mathrm{N}$ & $\mathrm{O}$ & * & $*$ & * & $*$ & $*$ & $*$ & * & * \\
\hline Senecio pinnatus Poir. & Hi $\mathrm{p}$ & $\mathrm{N}$ & PV & $*$ & $*$ & $*$ & $*$ & $*$ & $*$ & * & \\
\hline Soliva anthemifolia (Juss.) Sweet & Hi a & $\mathrm{N}$ & PV & & & $*$ & $*$ & * & & & \\
\hline Sonchus oleraceus $\mathrm{L}$. & Hi a & $\mathrm{E}$ & PV & & & $*$ & & $*$ & & & \\
\hline $\begin{array}{l}\text { Symphyotrichum squamatum } \\
\text { (Spreng.) G.L. Nesom }\end{array}$ & Hi $p$ & $\mathrm{~N}$ & $\mathrm{O}$ & $*$ & & & & $*$ & & & \\
\hline Taraxacum officinale F.H. Wigg. & Hi $\mathrm{p}$ & $\mathrm{E}$ & PV & & * & & & & & & \\
\hline $\begin{array}{l}\text { Urolepis hecatantha (DC.) R.M. } \\
\text { King \& H. Rob. }\end{array}$ & Hi a & $\mathrm{N}$ & $\mathrm{VO}$ & $*$ & & $*$ & $*$ & * & & & \\
\hline \multicolumn{12}{|l|}{ BIGNONIACEAE } \\
\hline $\begin{array}{l}\text { Amphilophium carolinae (Lindl.) } \\
\text { L.G. Lohmann }\end{array}$ & En $p$ & $\mathrm{~N}$ & VO & & $*$ & $*$ & & $*$ & $*$ & $*$ & $*$ \\
\hline \multicolumn{12}{|l|}{ BORAGINACEAE } \\
\hline Echium plantagineum $\mathrm{L}$. & Hi a-b & $\mathrm{E}$ & PV & & & & & * & & & \\
\hline Heliotropium curassavicum L. & Hi $p$ & $\mathrm{~N}$ & $\mathrm{~V}$ & & $*$ & & & & $*$ & $*$ & * \\
\hline \multicolumn{12}{|l|}{ BRASSICACEAE } \\
\hline $\begin{array}{l}\text { Capsella bursa-pastoris (L.) } \\
\text { Medik }\end{array}$ & Hi a & $\mathrm{E}$ & IP & & & & $*$ & & & & \\
\hline \multicolumn{12}{|l|}{ CACTACEAE } \\
\hline $\begin{array}{l}\text { Cleistocactus baumannii (Lem.) } \\
\text { Lem. }\end{array}$ & Su p & $\mathrm{N}$ & PV & & & & & & * & & \\
\hline Opuntia paraguayensis K. Schum. & Su p & $\mathrm{N}$ & PV & & & & & & $*$ & & \\
\hline
\end{tabular}


Apéndice. (Continuación). Lista florística de familias y especies por comunidades herbáceas. Se indica la forma de vida, origen y época de floración y fructificación.

\begin{tabular}{|c|c|c|c|c|c|c|c|c|c|c|c|}
\hline \multirow[b]{2}{*}{ Familia y especie } & \multirow[b]{2}{*}{$\begin{array}{l}\text { Forma } \\
\text { de vida }\end{array}$} & \multirow[b]{2}{*}{ Origen } & \multirow{2}{*}{$\begin{array}{l}\text { Floración } \\
\text { /fructif. }\end{array}$} & \multicolumn{8}{|c|}{ Comunidades y sus variantes } \\
\hline & & & & Flech. & $\begin{array}{l}\text { Flech. } \\
\text { Deg. }\end{array}$ & $\begin{array}{c}\text { Flech. } \\
\text { H. }\end{array}$ & P.H. & $\begin{array}{l}\text { P.H. } \\
\text { Deg. }\end{array}$ & Gram. & $\begin{array}{l}\text { Gram. } \\
\text { H. }\end{array}$ & $\begin{array}{l}\text { Gram. } \\
\text { Sal. }\end{array}$ \\
\hline \multicolumn{12}{|l|}{ CALYCERACEAE } \\
\hline Acicarpha tribuloides Juss. & Hi a & $\mathrm{N}$ & $\mathrm{V}$ & & & & $*$ & $*$ & & & \\
\hline \multicolumn{12}{|l|}{ CANNABACEAE } \\
\hline Celtis pallida Torr. & ar & $\mathrm{N}$ & PV & & & & & & & & $*$ \\
\hline Celtis tala Gillles ex Planch. & $\mathrm{Ar}$ & $\mathrm{N}$ & $\mathrm{P}$ & $*$ & $*$ & * & & $*$ & $*$ & $*$ & $*$ \\
\hline \multicolumn{12}{|l|}{ CARYOPHILLACEAE } \\
\hline Cerastium commersonianum DC. & Hi a & $\mathrm{N}$ & $\mathrm{V}$ & & & & $*$ & * & & & \\
\hline $\begin{array}{l}\text { Spergula platensis (Cambess.) } \\
\text { Shinners }\end{array}$ & Hi $\mathrm{p}$ & $\mathrm{N}$ & $\mathrm{P}$ & * & & & & & & & \\
\hline Stellaria media (L.) Cirillo & Hi a & $\mathrm{N}$ & $\mathrm{P}$ & & & & & * & & & \\
\hline \multicolumn{12}{|l|}{ CHENOPODIACEAE } \\
\hline Chenopodium album $\mathrm{L}$. & $\mathrm{Hi} \mathrm{a}$ & $\mathrm{E}$ & $\mathrm{V}$ & & $*$ & & & & & & \\
\hline $\begin{array}{l}\text { Dysphania ambrosioides (L.) } \\
\text { Mosyakin \& Clemants }\end{array}$ & Hi $\mathrm{p}$ & $\mathrm{N}$ & $\mathrm{V}$ & $*$ & $*$ & * & $*$ & * & & $*$ & \\
\hline \multicolumn{12}{|l|}{ CONVOLVULACEAE } \\
\hline Convolvulus hermanniae L`Hér. & En $p$ & $\mathrm{~N}$ & $\mathrm{~V}$ & & & $*$ & & & & & \\
\hline $\begin{array}{l}\text { Dichondra microcalyx (Hallier f.) } \\
\text { Fabris }\end{array}$ & Hi $\mathrm{p}$ & $\mathrm{N}$ & $\mathrm{P}$ & $*$ & $*$ & * & $*$ & $*$ & $*$ & $*$ & $*$ \\
\hline \multicolumn{12}{|l|}{ EUPHORBIACEAE } \\
\hline Euphorbia berteroana Spreng. & Hi a & $\mathrm{N}$ & PV & & & & $*$ & & & & \\
\hline $\begin{array}{l}\text { Sapium haematospermum Müll. } \\
\text { Arg. }\end{array}$ & $\mathrm{Ar}$ & $\mathrm{N}$ & PV & & $*$ & & & & & & \\
\hline \multicolumn{12}{|l|}{ FABACEAE } \\
\hline $\begin{array}{l}\text { Geoffroea decorticans (Gillies ex } \\
\text { Hook. \& Arn.) Burkart }\end{array}$ & $\mathrm{Ar}$ & $\mathrm{N}$ & PV & & $*$ & & & $*$ & $*$ & $*$ & * \\
\hline Melilotus albus Desr. & Hi a-b & $\mathrm{E}$ & $\mathrm{P}$ & & & & $*$ & & & & \\
\hline Parkinsonia aculeata $\mathrm{L}$. & $\mathrm{Ar}$ & $\mathrm{N}$ & PV & & $*$ & & $*$ & & & & \\
\hline Prosopis affinis Spreng. & $\mathrm{Ar}$ & $\mathrm{N}$ & PVO & $*$ & & & & & & & \\
\hline Prosopis alba Griseb. & $\mathrm{Ar}$ & $\mathrm{N}$ & PVO & $*$ & $*$ & $*$ & $*$ & $*$ & * & $*$ & $*$ \\
\hline $\begin{array}{l}\text { Senegalia praecox (Griseb.) } \\
\text { Seigler \& Ebinger }\end{array}$ & $\mathrm{Ar}$ & $\mathrm{N}$ & PVO & & $*$ & $*$ & $*$ & & $*$ & & \\
\hline Trifolium repens $\mathrm{L}$. & Hi $\mathrm{p}$ & $\mathrm{E}$ & PV & & & & $*$ & $*$ & & & \\
\hline
\end{tabular}


Apéndice. (Continuación). Lista florística de familias y especies por comunidades herbáceas. Se indica la forma de vida, origen y época de floración y fructificación.

\begin{tabular}{|c|c|c|c|c|c|c|c|c|c|c|c|}
\hline \multirow{2}{*}{ Familia y especie } & \multirow{2}{*}{$\begin{array}{l}\text { Forma } \\
\text { de vida }\end{array}$} & \multirow{2}{*}{ Origen } & \multirow{2}{*}{$\begin{array}{l}\text { Floración } \\
\text { /fructif. }\end{array}$} & \multicolumn{8}{|c|}{ Comunidades y sus variantes } \\
\hline & & & & Flech. & $\begin{array}{l}\text { Flech. } \\
\text { Deg. }\end{array}$ & $\begin{array}{l}\text { Flech. } \\
\text { H. }\end{array}$ & P.H. & $\begin{array}{l}\text { P.H. } \\
\text { Deg. }\end{array}$ & Gram. & $\begin{array}{l}\text { Gram. } \\
\text { H. }\end{array}$ & $\begin{array}{l}\text { Gram. } \\
\text { Sal. }\end{array}$ \\
\hline $\begin{array}{l}\text { Vachellia caven (Molina) Seigler } \\
\text { \& Ebinger }\end{array}$ & $\mathrm{Ar}$ & $\mathrm{N}$ & PVO & $*$ & $*$ & * & $*$ & * & & $*$ & \\
\hline \multicolumn{12}{|l|}{ GESNERIACEAE } \\
\hline Sinningia tubiflora (Hook.) Fritsch & Hi p & $\mathrm{N}$ & VO & & & & $*$ & & & & \\
\hline \multicolumn{12}{|l|}{ LAMIACEAE } \\
\hline $\begin{array}{l}\text { Cantinoa mutabilis (Rich.) Harley } \\
\& \text { J.F.B. Pastore }\end{array}$ & Hi a & $\mathrm{N}$ & $\mathrm{V}$ & * & $*$ & * & $*$ & $*$ & & $*$ & \\
\hline $\begin{array}{l}\text { Condea floribunda (Briq.) Harley } \\
\& \text { J.F.B. Pastore }\end{array}$ & Hi $\mathrm{p}$ & $\mathrm{N}$ & PV & & & & $*$ & & & & \\
\hline Scutellaria racemosa Pers. & Hi $\mathrm{p}$ & $\mathrm{N}$ & PV & & & $*$ & & $*$ & & & \\
\hline \multicolumn{12}{|l|}{ LYTHRACEAE } \\
\hline Heimia salicifolia (Kunth) Link & ar & $\mathrm{N}$ & PV & * & $*$ & * & $*$ & * & $*$ & * & \\
\hline \multicolumn{12}{|l|}{ MALVACEAE } \\
\hline $\begin{array}{l}\text { Callianthe pauciflora (A. St.- Hil.) } \\
\text { Dorr }\end{array}$ & ar & $\mathrm{N}$ & PV & & & * & $*$ & & & & \\
\hline $\begin{array}{l}\text { Modiolastrum gilliesii (Steud.) } \\
\text { Krapov. }\end{array}$ & Hi p & $\mathrm{N}$ & PV & & & & $*$ & $*$ & $*$ & $*$ & $*$ \\
\hline Sida rhombifolia L. & Hi p & $\mathrm{N}$ & $\mathrm{V}$ & $*$ & $*$ & $*$ & $*$ & $*$ & $*$ & $*$ & \\
\hline
\end{tabular}

MELIACEAE

Melia azedarach L.

Ar E PV

MORACEAE

Morus alba L.

Ar E PVO

OLEACEAE

Ligustrum lucidum W.T. Aiton

Ar $n \quad P V$

OXALIDACEAE

Oxalis corniculata L.

Hi p N P

PASSIFLORACEAE

Passiflora caerulea L.

En $\mathrm{p} \quad \mathrm{N} \quad \mathrm{PVO}$

PHYTOLACCACEAE

Rivina humilis L.

Hi p N VO

PLANTAGINACEAE 
Apéndice. (Continuación). Lista florística de familias y especies por comunidades herbáceas. Se indica la forma de vida, origen y época de floración y fructificación.

\begin{tabular}{|c|c|c|c|c|c|c|c|c|c|c|c|}
\hline \multirow[b]{2}{*}{ Familia y especie } & \multirow{2}{*}{$\begin{array}{l}\text { Forma } \\
\text { de vida }\end{array}$} & \multirow[b]{2}{*}{ Origen } & \multirow{2}{*}{$\begin{array}{l}\text { Floración } \\
\text { /fructif. }\end{array}$} & \multicolumn{8}{|c|}{ Comunidades y sus variantes } \\
\hline & & & & Flech. & $\begin{array}{l}\text { Flech. } \\
\text { Deg. }\end{array}$ & $\begin{array}{l}\text { Flech. } \\
\text { H. }\end{array}$ & P.H. & $\begin{array}{l}\text { P.H. } \\
\text { Deg. }\end{array}$ & Gram. & $\begin{array}{l}\text { Gram. } \\
\text { H. }\end{array}$ & $\begin{array}{l}\text { Gram. } \\
\text { Sal. }\end{array}$ \\
\hline Plantago myosuros Lam. & Hi a & $\mathrm{N}$ & PV & $*$ & $*$ & $*$ & $*$ & $*$ & $*$ & $*$ & \\
\hline Plantago tomentosa Lam. & Hi $\mathrm{p}$ & $\mathrm{N}$ & PV & & & & & $*$ & $*$ & $*$ & $*$ \\
\hline $\begin{array}{l}\text { Scoparia montevidensis (Spreng.) } \\
\text { R.E. Fr. }\end{array}$ & Hi $\mathrm{p}$ & $\mathrm{N}$ & $P$ & & $*$ & & & & & & \\
\hline \multicolumn{12}{|l|}{ POLYGALACEAE } \\
\hline $\begin{array}{l}\text { Polygala duarteana A. St.-Hil. } \\
\& \text { Moq. }\end{array}$ & Hi p & $\mathrm{N}$ & PV & & $*$ & & & & & & \\
\hline \multicolumn{12}{|l|}{ POLYGONACEAE } \\
\hline $\begin{array}{l}\text { Muehlenbeckia sagittifolia } \\
\text { (Ortega) Meisn. }\end{array}$ & En $p$ & $\mathrm{~N}$ & $\mathrm{VO}$ & & $*$ & * & $*$ & * & $*$ & & $*$ \\
\hline Polygonum punctatum Elliott & Hi a-p & $\mathrm{N}$ & $\mathrm{VO}$ & $*$ & & $*$ & $*$ & $*$ & & & \\
\hline Rumex crispus $\mathrm{L}$. & Hi $\mathrm{p}$ & $\mathrm{E}$ & PV & & & & $*$ & $*$ & & & \\
\hline \multicolumn{12}{|l|}{ PORTULACACEAE } \\
\hline Portulaca cryptopetala Speg. & Hi a-b & $\mathrm{N}$ & $\mathrm{V}$ & & $*$ & $*$ & & $*$ & & $*$ & $*$ \\
\hline Portulaca oleracea $\mathrm{L}$. & Hi a & $\mathrm{E}$ & $\mathrm{V}$ & $*$ & $*$ & $*$ & $*$ & $*$ & $*$ & $*$ & $*$ \\
\hline \multicolumn{12}{|l|}{ RANUNCULACEAE } \\
\hline Clematis montevidensis Spreng. & En $p$ & $\mathrm{~N}$ & PV & $*$ & $*$ & $*$ & $*$ & $*$ & & $*$ & \\
\hline \multicolumn{12}{|l|}{ RUBIACEAE } \\
\hline Rubiacea $\mathrm{sp}$. & & & & & $*$ & & $*$ & & & & \\
\hline
\end{tabular}

Buddleja stachyoides Cham. \& Schltdl.

ar $\mathrm{N} \quad \mathrm{P}$

\section{SOLANACEAE}

Cestrum parqui L'Hér.

Jaborosa integrifolia Lam.

Lycium boerhaviaefolium L. f.

Hi p N $\quad \mathrm{NO}$

Niembergia aristata D. Don

ar $\quad \mathrm{N}$

Physalis viscosa $\mathrm{L}$.

Hi p N PV

Salpichroa origanifolia (Lam.)

Baill.

Hi p N VO

Solanum pseudocapsicum L.

Hi p N PV

Hi p N PV 
Apéndice. (Continuación). Lista florística de familias y especies por comunidades herbáceas. Se indica la forma de vida, origen y época de floración y fructificación.

\begin{tabular}{|c|c|c|c|c|c|c|c|c|c|c|c|}
\hline \multirow[b]{2}{*}{ Familia y especie } & \multirow{2}{*}{$\begin{array}{l}\text { Forma } \\
\text { de vida }\end{array}$} & \multirow[b]{2}{*}{ Origen } & \multirow{2}{*}{$\begin{array}{l}\text { Floración } \\
\text { /fructif. }\end{array}$} & \multicolumn{8}{|c|}{ Comunidades y sus variantes } \\
\hline & & & & Flech. & $\begin{array}{l}\text { Flech. } \\
\text { Deg. }\end{array}$ & $\begin{array}{l}\text { Flech. } \\
\text { H. }\end{array}$ & P.H. & $\begin{array}{l}\text { P.H. } \\
\text { Deg. }\end{array}$ & Gram. & $\begin{array}{l}\text { Gram. } \\
\text { H. }\end{array}$ & $\begin{array}{l}\text { Gram. } \\
\text { Sal. }\end{array}$ \\
\hline Solanum pygmaeum Cav. & Hi $p$ & $\mathrm{~N}$ & PV & $*$ & $*$ & $*$ & $*$ & $*$ & & & $*$ \\
\hline Solanum sisymbriifolium Lam. & Hi p & $\mathrm{N}$ & VO & & & * & & * & & & $*$ \\
\hline \multicolumn{12}{|l|}{ TALINACEAE } \\
\hline $\begin{array}{l}\text { Talinum paniculatum (Jacq.) } \\
\text { Gaertn. }\end{array}$ & Hi p & $\mathrm{N}$ & PV & * & $*$ & $*$ & & $*$ & $*$ & & \\
\hline \multicolumn{12}{|l|}{ URTICACEAE } \\
\hline Parietaria debilis G. Forst. & Hi a & $\mathrm{E}$ & $\mathrm{P}$ & * & & $*$ & $*$ & $*$ & & & \\
\hline Urtica urens $\mathrm{L}$. & Hi a & $\mathrm{E}$ & IP & & $*$ & * & & $*$ & & & \\
\hline \multicolumn{12}{|l|}{ VERBENACEAE } \\
\hline $\begin{array}{l}\text { Aloysia gratissima (Gillies \& } \\
\text { Hook. ex Hook.) Tronc. }\end{array}$ & ar & $\mathrm{N}$ & PV & & & & $*$ & & & & \\
\hline $\begin{array}{l}\text { Glandularia tweedieana (Niven ex } \\
\text { Hook.) P. Peralta }\end{array}$ & Hi p & $\mathrm{N}$ & PV & & & & $*$ & $*$ & & & \\
\hline $\begin{array}{l}\text { Verbena intermedia Gillies \& } \\
\text { Hook. ex Hook. }\end{array}$ & Hi $\mathrm{p}$ & $\mathrm{N}$ & IPV & * & $*$ & * & $*$ & $*$ & & & \\
\hline
\end{tabular}

\section{MONOCOTILEDONEAS}

\section{ALISMATACEAE}

Sagittaria montevidensis Cham. \& Schltdl.

Hi p N PV

\section{BROMELIACEAE}

Tillandsia aëranthos (Loisel.)

L.B. Sm.

Ep p N PV *

Tillandsia recurvata (L.) L.

Ep p N PV

\section{COMMELINACEAE}

Commelina erecta $\mathrm{L}$.

Hi p N $\quad \mathrm{V}$

CYPERACEAE

Carex bonariensis Desf. ex Poir.

Hi p N V

Carex sp.

Hi p

Cyperus entrerianus Boeck.

Hi p N PV

Cyperus rotundus $\mathrm{L}$.

Hi p

Cyperus sesquiflorus (Torr.) Mattf. \& Kük. ex Kük.

Hi p N V


Apéndice. (Continuación). Lista florística de familias y especies por comunidades herbáceas. Se indica la forma de vida, origen y época de floración y fructificación.

\begin{tabular}{|c|c|c|c|c|c|c|c|c|c|c|c|}
\hline \multirow[b]{2}{*}{ Familia y especie } & \multirow{2}{*}{$\begin{array}{l}\text { Forma } \\
\text { de vida }\end{array}$} & \multirow[b]{2}{*}{ Origen } & \multirow{2}{*}{$\begin{array}{l}\text { Floración } \\
\text { /fructif. }\end{array}$} & \multicolumn{8}{|c|}{ Comunidades y sus variantes } \\
\hline & & & & Flech. & $\begin{array}{l}\text { Flech. } \\
\text { Deg. }\end{array}$ & $\begin{array}{l}\text { Flech. } \\
\text { H. }\end{array}$ & P.H. & $\begin{array}{l}\text { P.H. } \\
\text { Deg. }\end{array}$ & Gram. & $\begin{array}{l}\text { Gram. } \\
\text { H. }\end{array}$ & $\begin{array}{l}\text { Gram. } \\
\text { Sal. }\end{array}$ \\
\hline Cyperus virens Michx. & Hi p & $\mathrm{N}$ & V & & $*$ & * & $*$ & & $*$ & $*$ & $*$ \\
\hline $\begin{array}{l}\text { Eleocharis elegans (Kunth) Roem. } \\
\& \text { Schult. }\end{array}$ & Hi $p$ & $\mathrm{~N}$ & $\mathrm{~V}$ & & & $*$ & & * & & & \\
\hline Eleocharis flavescens (Poir.) Urb. & Hi $\mathrm{p}$ & $\mathrm{N}$ & V & $*$ & $*$ & & $*$ & $*$ & $*$ & $*$ & \\
\hline $\begin{array}{l}\text { Fimbristilis autumnalis (L.) } \\
\text { Roem. \& Schult. }\end{array}$ & Hi p & $\mathrm{N}$ & V & & & & & & $*$ & $*$ & \\
\hline IRIDACEAE & & & & & & & & & & & \\
\hline Cypella herbertii (Lindl.) Herb. & Hi $\mathrm{p}$ & $\mathrm{N}$ & $\mathrm{P}$ & $*$ & $*$ & & $*$ & & & & \\
\hline $\begin{array}{l}\text { Herbertia lahue (Molina) } \\
\text { Goldblatt }\end{array}$ & Hi a & $\mathrm{N}$ & $\mathrm{P}$ & $*$ & $*$ & $*$ & & $*$ & & $*$ & \\
\hline Sisyrinchum pachyrhizum Baker & Hi p & $\mathrm{N}$ & PV & & $*$ & & & & & & \\
\hline
\end{tabular}

\section{JUNCACEAE}

Juncus bufonius L.

Hi a N PV

\section{POACEAE}

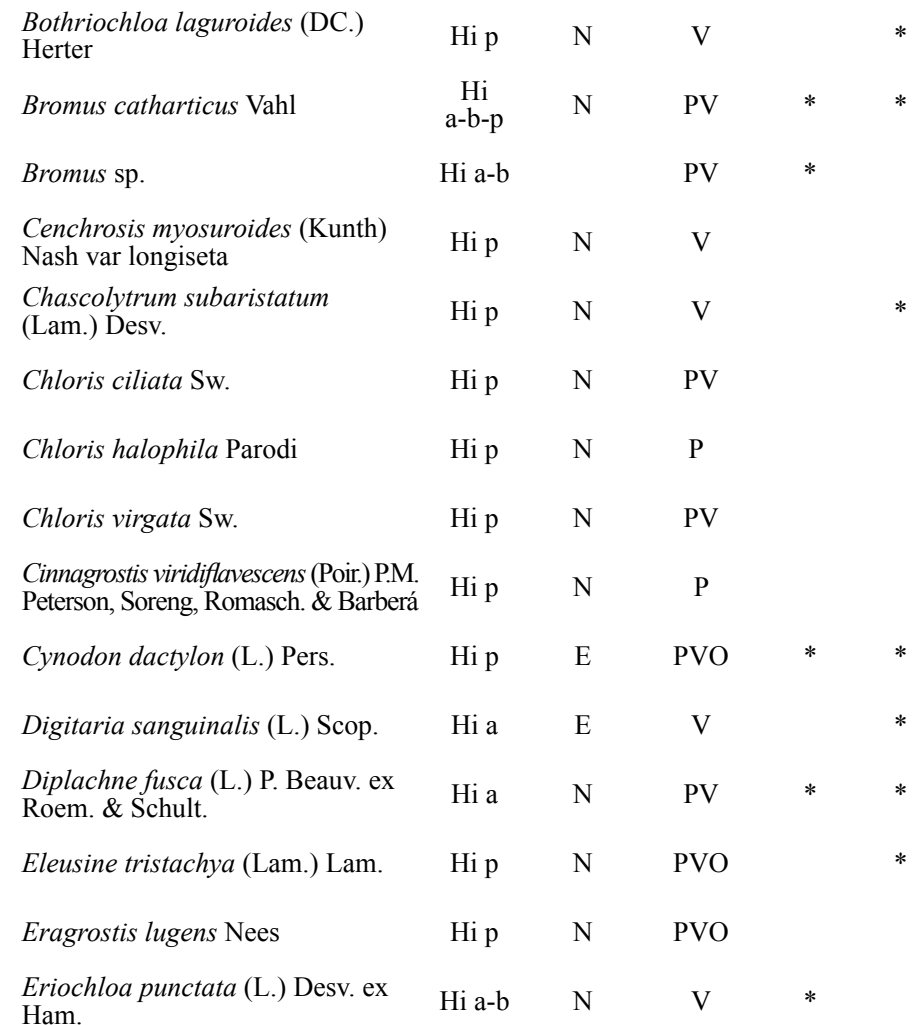




\section{P. A. CAPELINO \& A. G. BENDER. Vegetación herbácea Espinal}

Apéndice. (Continuación). Lista florística de familias y especies por comunidades herbáceas. Se indica la forma de vida, origen y época de floración y fructificación.

\begin{tabular}{|c|c|c|c|c|c|c|c|c|c|c|c|}
\hline \multirow[b]{2}{*}{ Familia y especie } & \multirow{2}{*}{$\begin{array}{l}\text { Forma } \\
\text { de vida }\end{array}$} & \multirow[b]{2}{*}{ Origen } & \multirow{2}{*}{$\begin{array}{l}\text { Floración } \\
\text { /fructif. }\end{array}$} & \multicolumn{8}{|c|}{ Comunidades y sus variantes } \\
\hline & & & & Flech. & $\begin{array}{l}\text { Flech. } \\
\text { Deg. }\end{array}$ & $\begin{array}{l}\text { Flech. } \\
\text { H. }\end{array}$ & P.H. & $\begin{array}{l}\text { P.H. } \\
\text { Deg. }\end{array}$ & Gram. & $\begin{array}{l}\text { Gram. } \\
\text { H. }\end{array}$ & $\begin{array}{c}\text { Gram. } \\
\text { Sal. }\end{array}$ \\
\hline $\begin{array}{l}\text { Eustachys distichophylla (Lag.) } \\
\text { Nees }\end{array}$ & Hi p & $\mathrm{N}$ & PV & & & & & & & $*$ & $*$ \\
\hline Hordeum stenostachys Godr. & Hi p & $\mathrm{N}$ & PV & & & & & & $*$ & $*$ & $*$ \\
\hline Leersia hexandra Sw. & Hi p & $\mathrm{N}$ & $\mathrm{PV}$ & $*$ & * & & & * & & & \\
\hline Lolium multiflorum Lam. & Hi a & $\mathrm{E}$ & PV & $*$ & & & & & & & \\
\hline Melica argyrea Hack. & Hi $\mathrm{p}$ & $\mathrm{N}$ & $\mathrm{P}$ & & & & $*$ & $*$ & $*$ & $*$ & $*$ \\
\hline Melica macra Ness & Hi p & $\mathrm{N}$ & $\mathrm{P}$ & $*$ & $*$ & & $*$ & $*$ & & & \\
\hline $\begin{array}{l}\text { Nassella hyalina (Ness) } \\
\text { Barkworth }\end{array}$ & Hi $p$ & $\mathrm{~N}$ & $\mathrm{P}$ & $*$ & $*$ & $*$ & $*$ & $*$ & $*$ & $*$ & $*$ \\
\hline $\begin{array}{l}\text { Nassella neesiana (Trin. \& Rupr.) } \\
\text { Barkworth }\end{array}$ & Hi $\mathrm{p}$ & $\mathrm{N}$ & $\mathrm{P}$ & $*$ & $*$ & $*$ & $*$ & $*$ & $*$ & $*$ & $*$ \\
\hline Panicum bergii Arechav. & Hi $\mathrm{p}$ & $\mathrm{N}$ & PV & & & & & & $*$ & $*$ & \\
\hline Paspalum dilatatum Poir. & Hi $\mathrm{p}$ & $\mathrm{N}$ & PV & & & & & $*$ & $*$ & $*$ & $*$ \\
\hline Paspalum notatum Flüggé & Hi $\mathrm{p}$ & $\mathrm{N}$ & $\mathrm{V}$ & & & & $*$ & $*$ & $*$ & & \\
\hline $\begin{array}{l}\text { Paspalum unispicatum (Scribn. \& } \\
\text { Merr.) Nash }\end{array}$ & Hi $\mathrm{p}$ & $\mathrm{N}$ & $\mathrm{V}$ & & $*$ & & & & & & $*$ \\
\hline Phalaris angusta Nees ex Trin. & $\begin{array}{c}\mathrm{Hi} \\
\mathrm{a}-\mathrm{b}-\mathrm{p}\end{array}$ & $\mathrm{N}$ & PV & $*$ & $*$ & & $*$ & $*$ & * & $*$ & \\
\hline $\begin{array}{l}\text { Piptochaetium stipoides (Tirn. \& } \\
\text { Rupr.) Hack. }\end{array}$ & Hi $\mathrm{p}$ & $\mathrm{N}$ & PV & $*$ & $*$ & $*$ & $*$ & & $*$ & $*$ & * \\
\hline Poa annua L. & Hi a & $\mathrm{E}$ & $\mathrm{P}$ & & & $*$ & & & & & \\
\hline Poa bonariensis (Lam.) Kunth & Hi $\mathrm{p}$ & $\mathrm{N}$ & $\mathrm{P}$ & $*$ & & & & & & & \\
\hline Poa lanigera Nees & Hi $\mathrm{p}$ & $\mathrm{N}$ & $\mathrm{P}$ & & & & $*$ & & & & \\
\hline Setaria fiebrigii R.A.W. Herrm. & Hi $p$ & $\mathrm{~N}$ & PV & $*$ & & $*$ & $*$ & $*$ & & & \\
\hline $\begin{array}{l}\text { Setaria geminata (Forssk.) } \\
\text { Veldkamp }\end{array}$ & Hi $p$ & $\mathrm{~N}$ & PV & & $*$ & $*$ & & $*$ & & $*$ & \\
\hline $\begin{array}{l}\text { Setaria parviflora (Poir.) } \\
\text { Kerguélen }\end{array}$ & Hi p & $\mathrm{N}$ & $\mathrm{VO}$ & $*$ & $*$ & & $*$ & $*$ & $*$ & $*$ & * \\
\hline Sporobolus indicus (L.) R. Br. & Hi $p$ & $\mathrm{~N}$ & $\mathrm{~V}$ & & & & & & $*$ & $*$ & \\
\hline $\begin{array}{l}\text { Sporobolus pyramidatus (Lam.) } \\
\text { Hitchc. }\end{array}$ & Hi p & $\mathrm{N}$ & PV & & $*$ & & & & $*$ & $*$ & $*$ \\
\hline Steinchisma hians (Elliott) Nash & Hi $\mathrm{p}$ & $\mathrm{N}$ & PV & & $*$ & $*$ & * & $*$ & $*$ & $*$ & $*$ \\
\hline
\end{tabular}

Forma de vida: Ar: árbol; ar: arbusto; En a: enredadera anual; En p: enredadera perenne; Hp: epífita; Hi a: hierba anual; Hi a-b: hierba anual-bianual; Hi a-b-p: hierba anual-bianual o perenne. Su p: suculenta perenne. Origen: N: nativa; E: exótica. Floración-fructificación: P: primavera; V: verano. O: otoño. I: invierno. Comunidades: Flech.: flechillar; Flech. Deg.: fechillar degradado; Flech. H.: flechillar húmedo. Gram.: gramillar; Gram. H.: gramillar húmedo. Gram. Sal.: gramillar salino. P.H.: pradera húmeda; P.H. Deg.: pradera húmeda degradada. * indica presencia de la especie. 